Randomised controlled trial

\title{
Benefits of nurse-led cardiovascular prevention for patients with type 1 diabetes
}

\section{Helene Voogdt-Pruis, ${ }^{1}$ H J M Vrijhoef, ${ }^{1,2}$ G H M I Beusmans ${ }^{1}$}

10.1136/ebn.2011.100051

${ }^{1}$ Department of Integrated Care, School for Public Health and Primary Care (CAPHRI), Maastricht University Medical Centre, Maastricht, The Netherlands

${ }^{2}$ TRANZO, University of Tilburg, Tilburg, The Netherlands

Correspondence to Helene Voogdt-Pruis Department of Integrated Care, School for Public Health and Primary Care (CAPHRI), Maastricht University Medical Centre, Loc Mecc-N1 TransmZ, PO Box 5800, Maastricht 6202 AZ, The Netherlands; hrvoogdt@gmail.com

Commentary on: Wallymahmed ME, Morgan C, Gill GV, et al. Nurse-led cardiovascular risk factor intervention leads to improvements in cardiovascular risk targets and glycaemic control in people with type 1 diabetes when compared with routine diabetes clinic attendance. Diabet Med 2011;28:373-9.

\section{Implications for practice and research}

- Nurse-led cardiovascular prevention for patients with type 1 diabetes is effective because of an increased use of medication.

- Quality improvement of nurse-led prevention is needed to address unhealthy lifestyles.

- Future studies need to pay attention to the impact on quality of life and cost-effectiveness.

\section{Context}

Owing to the increasing ageing of the population and other factors, health services are confronted with increasing demands. Task substitution from doctors to nurses is one of the options to meet with these challenges. As nurses are accustomed to working with protocols, they might be better at adhering to guidelines and therefore be important for quality improvement of care. Studies have shown task substitution from doctors to nurses to be clinically effective. ${ }^{1}$ ${ }^{2}$ Wallymahmed and colleagues investigated the effectiveness of a nurse-led cardiovascular prevention programme to patients with type 1 diabetes attending a Diabetes Centre in Liverpool. Within the Diabetes Centre, treatment targets on cardiovascular risk factors turned out to be difficult to achieve. Nurse-led care was expected to be more effective.

\section{Methods}

For this study, the researchers randomised 81 type 1 diabetes patients to either usual care $(n=40)$ or nurse-led care $(n=41)$. Usual care involved review by doctors in a diabetes clinic with follow-up and referral to the multidisciplinary team for diabetes control problems. One specialised nurse was involved in nurse-led care. Over a period of 2 years, patients were reviewed four times within usual care and nine times within nurse-led care. Risk factors were measured three times within usual care and four times within nurse-led care.

\section{Findings}

Within nurse-led care, significant improvements in $\mathrm{HbA1c}$, total cholesterol and blood pressure were found. Within usual care, only total cholesterol improved. No significant changes in body mass index (BMI), weight or daily insulin dose were observed in both groups. More patients in both groups were 
taking lipid-lowering and antihypertensive agents' medication at the end than at the baseline. The authors concluded improvements within nurse-led care were probably attributable to the increased use of medication.

\section{Commentary}

The current study adds evidence to support the involvement of nurses in cardiovascular prevention. ${ }^{2}$ Long-term improvements in most risk factors within nurse-led care were remarkable. However, the study has some limitations in terms of its external validity: only one nurse was involved in nurse-led care and the remarkable decrease in risk factors could be due to her individual experience and effort (nine visits!). In addition, this nurse was not blinded for study outcome; hence, the possibility must be taken into account that this nurse performed well because of the awareness that her performance was being assessed. Therefore, external validity of this study is low.

At baseline, mean blood pressure was very low and SD was higher than expected. Apparently, a small portion of patients had very high blood pressure. Still, a high increase in use of medication took place with additional negative side effects of using medication. Should young patients with a slightly elevated risk factor be treated with medicine? These young patients with type 1 diabetes (mean=35 years) were overweight (mean BMI=28). No changes in BMI and weight could be found during 2 years of follow-up. It would have been better to focus on lifestyle changes: being active, changing diet - eating fish will have a positive impact on high-density lipoprotein too - and quitting smoking. Although lifestyle changes are difficult to achieve, results of nurse-led lifestyle interventions are promising. ${ }^{3}$
The benefits of nurse-led prevention in patients with type 1 diabetes are expected to be different within clinical practice. Continuous quality improvement strategies are needed for better implementation of nurse-led care. According to another study, nurses and doctors should inquire about patient adherence on a regular basis. In addition, practical skills in motivational interviewing and lifestyle interventions should be enhanced. ${ }^{4}$ In particular for young adults with type 1 diabetes, electronic self-management programmes on diet and physical exercise could be very effective, also in order to improve the attendance rate of patients to treatment policies. Given the reasons to start such nurse-led interventions, future studies need to pay attention to the impact on quality of life and cost-effectiveness.

\section{Competing interests None.}

\section{References}

1. Laurant M, Reeves D, Hermens R, et al. Substitution of doctors by nurses in primary care. Cochrane Database Syst Rev 2005;2:CD001271.

2. Voogdt-Pruis HR, Beusmans GH, Gorgels AP, et al. Effectiveness of nurse-delivered cardiovascular risk management in primary care: a randomised trial. Br J Gen Pract 2010;60:40-6.

3. Berra K. Does nurse case management improve implementation of guidelines for cardiovascular disease risk reduction? J Cardiovasc Nurs 2011;26:145-67.

4. Voogdt-Pruis HR, Van Ree JW, Gorgels AP, et al. Adherence to a guideline on cardiovascular prevention: a comparison between general practitioners and practice nurses. Int J Nurs Stud 2011;48:798-807. 